\title{
Metrology and ergonomic mensuration parameters
}

\author{
Cardoso, $\mathrm{C}^{\mathrm{a}}$ and Drucker, $\mathrm{L}^{\mathrm{a}}$ \\ ${ }^{a}$ National Institute of Metrology, Quality and Technology, Inmetro, Av. N. Sra. Das Graças, 50, Rio de Janeiro, \\ Brazil.E-mails: cacardoso@inmetro.gov.br and lpdrucker@inmetro.gov.br
}

\begin{abstract}
This poster intends to show how measurement, its concepts and methods are decisive to the ergonomic praxis and why their agents should be aware of how incertainty can be taken in the process in order to increase the accuracy of measurement and findings.
\end{abstract}

Keywords: measurement; uncertainty; standartization

\section{Introduction}

The Pysical ergonomics focuses on evaluating work conditions in order to adjust them to human needs, overall related with environmental comfort and usability of biomechanical devices. When we focus on a specific work station, a case-to-case approach is desirable, in spite the use of same analitical tools, we can consider some variations on the analyses results.

Ergonomic considerations enter the picture after measurement of a work station, according to parameters, shows that it needs to be rebuilt in a human scale. Such parameters are defined as measurement units within an interval of minimal and maximal values useful to decision process. The metric system, which units are based on the decimal number scale, was established in 1799 in Europe, in order to standardize measurement in a universally accessible way.

Later with the need to encompass all physical material properties, were defined other standards that became known as The International System of Units (SI), composed by seven bases of units: meter, kilogram, second, ampere, degree Kelvin (later renamed the kelvin), candela and mole. From those units we have the also called derived units.

\section{Discussion}

We are pointing out inside the ergonomic scope of the NR-17, the Brazilian Work Condition Regulamentation, and the importance of mesuration parameters as Lux, Celsius, Centimeters and Angles. To get to know the work conditions and to decide any inter- vention on it, it is necessary to measure those parameters and is also so important to know about measurement science.

In order to learn about mesuration science it is mandatory to understand some mathematical concept, some statistical tools, as well as operational measurement processes It is also advisable to learn some physical notions that help us define quantities. Is also important to realize the difference between the definition of a unit and its realisation. The definition of each base unit of the SI is drawn up so that it is unique and provides a sound theoretical basis upon which the most accurate and reproducible measurements can be made. The realisation of the definition of a unit is the procedure by which the definition may be used to establish the value and associated uncertainty of a quantity of the same kind as the unit.

Common sense believes that each physical quantity has a precise value that is what in metrology is called the "true quantity value". When making continuous measurements we realize different results to one same quantity and rarely we can repeat the same set of values in a repeated mesuration process. This fact reveals the impossibility to get to know the true value of one physical quantity. This is why we need to introduce here the measurement uncertainty concept. The measurement uncertainty is by definition the parameter associated to the results of one measurement, and it is related to the values dispersion which can be associated to what is being measured. That way, in order to decrease the uncertain relative to the measurement process statistical tools such as standard deviation, degree of confidence, probability distributions and error definition are useful. 


\section{Findings}

Every measurement has its outcome influenced by the way how the actions of agents such as metrological measurement method, the sample, the operator, the measuring equipment, the environmental conditions and traceability of equipment are managed.

In order to understand such afore mentioned concepts as measurement uncertainty and the impossibility to reach a true value in measurement process, we must understand the nature of measurement errors.

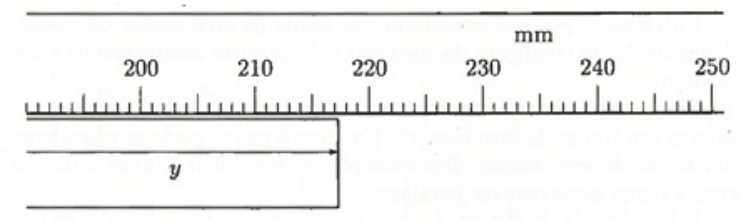

Figure 1: Millimeters rule graduate reading. [1] pp.133)

If we use a rule to measure some object represented Figure 1 as the "y" we can reach different results in a successive measurement once the result can be situated inter space of scale. This imprecision can be interpreted as 218,5 or 218,4 and so on, same if you have a smaller rule graduation you always we have some interstitial space between scales and it is called "imprecision".

We never can realize a "true value" or a "perfect value" but a "conventional value". It is described as imprecision related to the measurement reader is also know as measurement errors.

Measurement errors can be classified as systematic and random. Systematic errors generate deviation related to true value always in same pattern for plus or for less and far from random factors it can be generate by measurement instrument error due bad of instrumental calibration; environmental error are due to interference of environmental factors such as temperature, pressure, and the earth's magnetic field, which can cause experience interference; reading error to which we had already related and also caused by parallax (when the measurement process is made by a pointer instrument) Finally, the theoretical error due to indirect formula measure use and physical constant approximate values.

Random errors happens in the course of a number of measurement of same measurand and varies in an unpredictable way. It can be exemplified by variation occurs weighting process when the surface vibration under the weighting instrument. The Chapanis illustration [2] (Figure 2), using the example of a rifleman shooting at target shows the different error pattern. In the " $\mathrm{A}$ " target, the cluster of shots is centered on bull-eye, but widely dispersed is the good random error representation, with small mean error, large standard deviation. The B target, is a typical systematic error representation, the cluster shots is not centered on the bull-eye, but they are all close to another, that show a large mean error with a small standard deviation.

Non-calibrated measurement instruments can develop systematic errors which can be easily detectable.
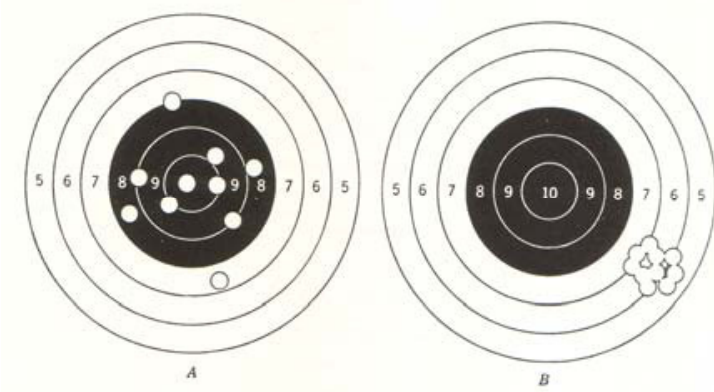

Figure 2: Random and systematic error illustration [2]. pp.52

\section{Conclusion}

Errors are part of the production of uncertainty of measurement, which means doubtfulness about the validity of the result of a measurement, and it characterizes the dispersion on the values that could be reasonable be attributed to the mesurand. (VIM).

To increase the accuracy of measurement results we must estimate a correction value of the mesurand through some statistical formulas and aggregate it on successive measurement.

In short, the ergonomic practices are very impacted by the ways that measurement is taken. The ergonomist should know the metrological subtleness's in order to achieve a better accuracy.

\section{References}

[1] Vuolo, José Henrique. Fundamentos da Teoria de Erros. São Paulo, Ed. Edgard Blucher Ltda. 1996.

[2] Chapanis, Alphonse. The Chapanis Chronicles: 50 Years of Human Factors Research, Education, and Design. Santa Barbara, Aegean Publishing Company. 1999. 